\title{
Leiomiossarcoma mixoide de vagina: relato de caso
}

\section{Vagina myxoid leyomiosarcoma: case report}

Camila Flávia Uchôa Alexandre1. Raquel Autran Coelho Peixoto². Karla Maria Rêgo Leopoldo Melo², Diane Isabelle Magno Cavalcante ${ }^{1}$.

1 Maternidade Escola Assis Chateaubriand (MEAC), Fortaleza, Ceará, Brasil. 2 Universidade Federal do Ceará (UFC), Fortaleza, Ceará, Brasil. 3 Hospital Geral de Fortaleza (HGF), Fortaleza, Ceará, Brasil.

\section{RESUMO}

As lesões malignas primárias da vagina são incomuns e os sarcomas vaginais são ainda mais raros. leiomissosarcomas são tumores que se originam do musculo liso. Descrevemos um caso raro de leiomiossarcoma mixoide de vagina com proposta inicial de tratamento cirúrgico. Uma mulher de 31 anos com vida sexual ativa apresentou massa vaginal, cujo exame histopatológico revelou leiomiossarcoma mixoide vagina sendo necessário estudo imunohistoquímico complementar que confirmou o diagnóstico. A paciente foi submetida a cirurgia para exérese da lesão, com bom resultado. A paciente permanece em acompanhamento no ambulatório de cirurgia oncológica, e apresenta-se sem recidiva de doença. A experiência com leiomiossarcomas vaginais é limitada. Os métodos ótimos de tratamento ainda não foram estabelecidos devido à raridade do tumor.

Palavras-chave: Leiomiossarcoma. Neoplasias vaginais. Oncologia cirúrgica.

\section{ABSTRACT}

Primary malignant lesions of the vagina are uncommon and vaginal sarcomas are even more rare. leiomyosarcomas are tumors that originate from smooth muscle. We describe a rare case of myxoid leiomyosarcoma of the vagina with initial proposal a surgical treatment. A 31-year-old woman, with an active sex life, presented a vaginal mass, whose histopathological examination revealed myxoid leiomyosarcoma in the vagina, requiring a complementary immunohistochemical study that confirmed the diagnosis. The patient underwent surgery for excision of the lesion, with good results. The patient remains in follow-up at the oncology surgery outpatient clinic, and has no disease recurrence. Experience with vaginal leiomyosarcomas is limited. Optimal treatment methods have not yet been established due to the rarity of the tumor.

Keywords: Leiomyosarcoma. Vaginal neoplasm. Surgical oncology.

Autor correspondente: Camila Flávia Uchôa Alexandre, Rua Zildenia, 749, Bairro Amador, Eusebio, Ceará. CEP: 61760-000. Telefone: +55 85 99959-7090. E-mail: cfualexandre@gmail.com

Conflito de interesses: Não há qualquer conflito de interesses por parte de qualquer um dos autores.

Recebido em: 28 Jun 2020; Revisado em: 12 Mar 2021; Aceito em: 15 Mar 2021. 


\section{INTRODUÇÃO}

Os carcinomas primários da vagina são raros, representando menos de $2 \%$ das neoplasias ginecológicas. O tipo histológico mais comum é o carcinoma epidermoide (90\%), seguido por adenocarcinoma (5-10\%), melanoma (3\%) e sarcomas (menos de $3 \%) .{ }^{1}$ O leiomiossarcoma é o tipo mais comum de sarcoma vaginal em mulheres adultas, originado de células do músculo liso. Pode atingir grandes tamanhos e, algumas vezes, ulcerar. ${ }^{2}$

No quadro clínico, o sintoma mais frequente é o sangramento vaginal. Pode ocorrer ainda corrimento, dispareunia, sinusiorragia e a presença de tumor vegetante no introito vaginal. $^{2}$

O tratamento ainda é controverso. Não há estudos mostrando superioridade na sobrevida de pacientes que são submetidas a cirurgia e quimioterapia/radioterapia sobre as pacientes que são submetidas apenas a cirurgia. ${ }^{3}$ A literatura ainda é escassa. Aqui, descrevemos um caso de leiomiossarcoma da vagina com tratamento cirúrgico.

\section{METODOLOGIA}

As informações contidas neste trabalho foram obtidas por meio de revisão do prontuário, após a aprovação pelo comitê de ética, além de revisão de literatura.

\section{DESCRIÇÃO DO CASO}

Mulher de 31 anos, nuligesta, com vida sexual ativa, apresentou queixa de sangramento transvaginal acíclico iniciado em fevereiro de 2019. Nunca havia realizado Papanicolau. O exame pélvico revelou uma massa globular de superfície lisa com origem em parede vaginal anterior/ lateral no terço médio, de aproximadamente $5 \times 6 \mathrm{~cm}$. O colo do útero estava normal. Não foram palpados linfonodos pélvicos ou inguinais. $\mathrm{O}$ exame retal não revelou nenhuma anormalidade. Uma primeira biópsia ambulatorial evidenciou processo inflamatório crônico acentuado. Foi realizada biópsia excisional em centro cirúrgico. Cistoscopia descartou invasão da bexiga. O histopatológico mostrou lesão fusocelular de baixo grau, infiltrativa e mixoide de vagina, podendo corresponder a leiomiossarcoma mixóide. Estudo imunohistoquimico mostrou-se compatível com leiomiossarcoma mixoide de baixo grau, com diferenciação presente, atividade mitótica ausente, necrose presente em menos de $50 \%$ da peça, atipias leves presentes e bordas de crescimento infiltrativo (Figuras 1, 2, 3 e 4).

Ressonância Magnética de pelve foi realizada e evidenciou nódulo vaginal endofítico, determinando aparente deiscência de parede vaginal no espaço vesico-uterino medindo 4,3 x 4,5 x 4,2 cm, além de lesão em região anexial esquerda sugestiva de endometrioma (Figuras 5).

Foi decidido por realizar exérese de massa vaginal com margens (Figura 6). O procedimento foi realizado sem intercorrências, com exérese total do tumor vaginal. A peça cirúrgica foi submetida a exame histopatológico e em primeira avaliação foi classificada como angiomixoma de parede vaginal, porém, ao exame imunohistoquimico, foi corroborado o anterior diagnostico de leiomiossarcoma mixoide de baixo grau. Paciente em acompanhamento no ambulatório, sem evidências de recidiva da doença até o momento.

Figura 1. Corte histológico exibindo grande quantidade de material mixoide e numerosos vasos de pequeno calibre.

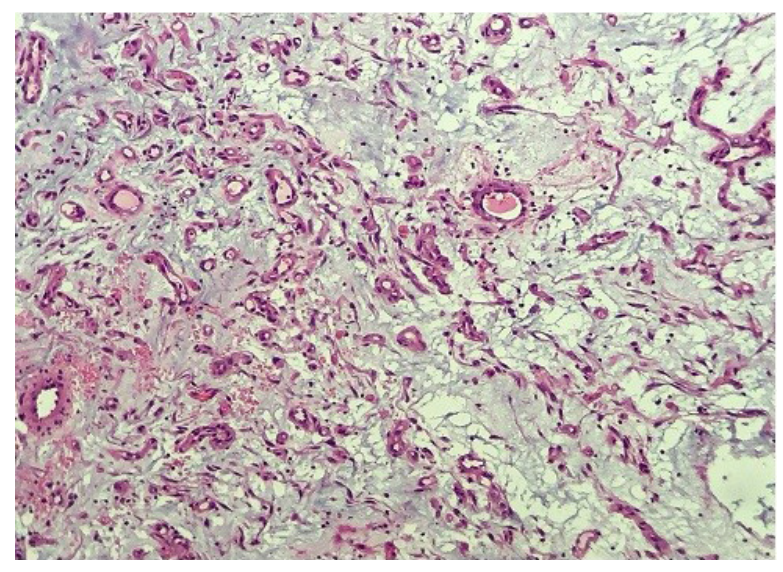

Figura 2. Corte histológico exibindo células fusiformes, de citoplasma alongado.

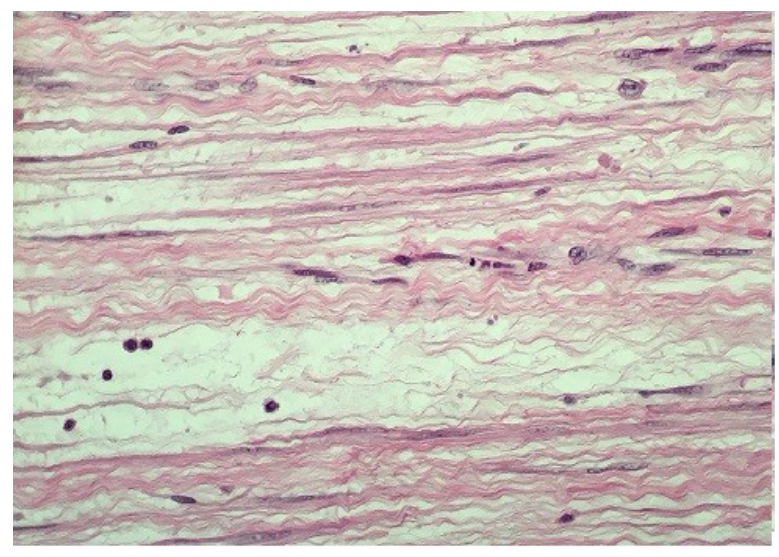

Figura 3. Imuno-histoquímica evidenciando positividade para marcador EMA.

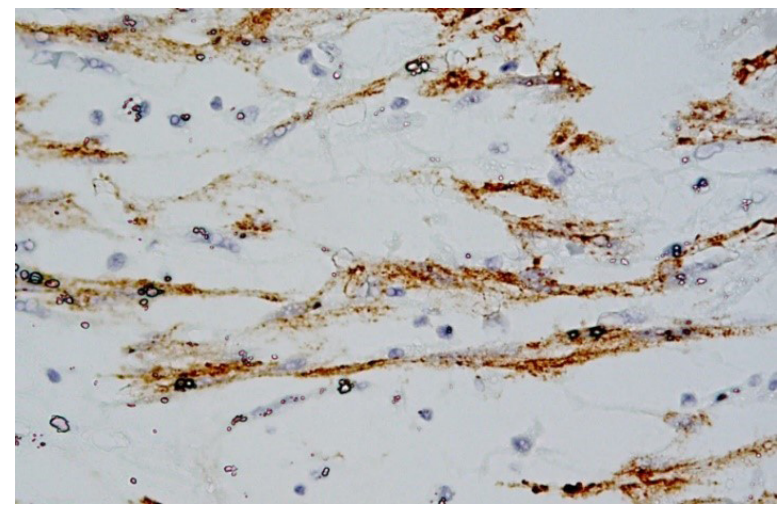


Figura 4. Imuno-histoquímica evidenciando positividade para actina músculo-específica.

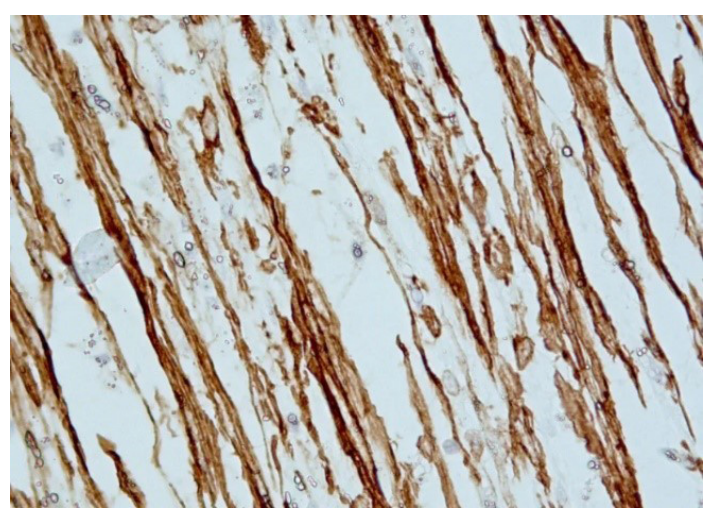

Figura 5. Imagem de ressonância de pelve.

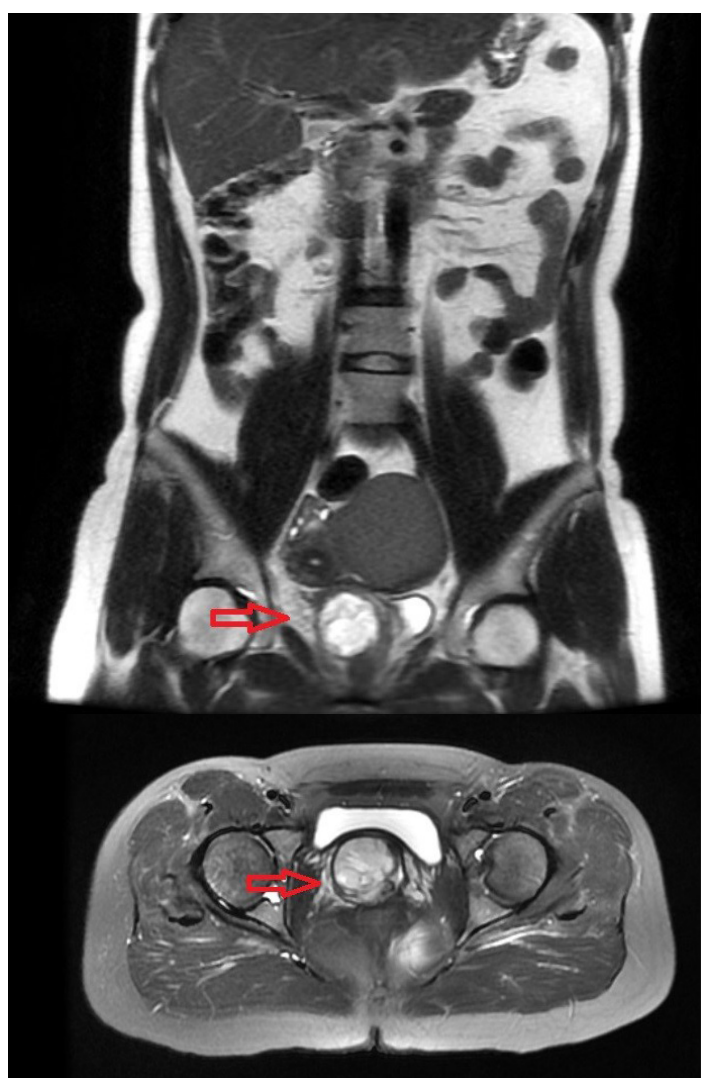

Figura 6. Peça cirúrgica resultado de ressecção total do tumor.

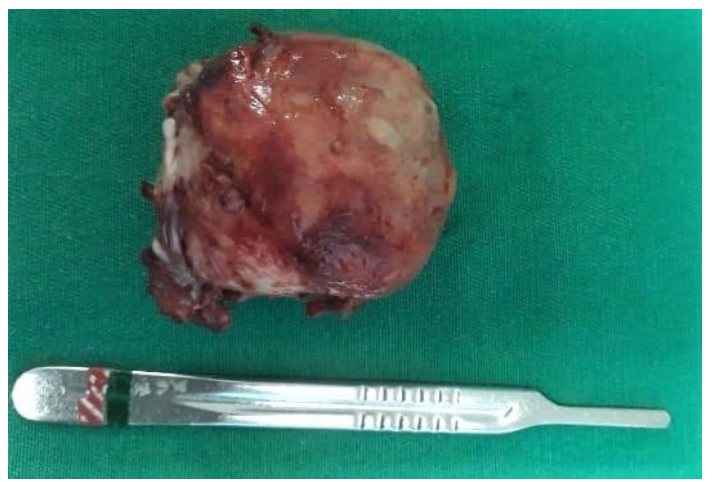

\section{DISCUSSÃO}

Leiomiossarcomas vaginais representam menos de $1 \%$ dos tumores pélvicos femininos, porém, são os tumores mesenquimais mais comuns em mulheres adultas. Os leiomiossarcomas mixoides são definidos como neoplasias que contêm grande quantidade de estroma de mixoide. ${ }^{1} \mathrm{O}$ estadiamento FIGO para carcinoma vaginal concentra-se no tamanho e profundidade da invasão; no entanto, não há estadiamento formal estabelecido para o leiomiossarcoma vaginal. ${ }^{1}$ Os critérios utilizados para diagnosticar tumores do músculo liso incluem a presença de atipia citológica moderada a acentuada, atividade mitótica aumentada (pelo menos 5 mitoses/10HPF (High Power Field)), necrose tumoral coagulativa e margens infiltrativas. A imuno-histoquímica revela marcadores como actina e desmina, fortes marcadores de neoplasia de musculo liso. ${ }^{2}$

Geralmente, pacientes com essa neoplasia apresentam massa vaginal assintomática. Em outros casos, como na paciente descrita, corrimento vaginal ou sangramento podem estar presentes. ${ }^{3}$

O leiomiossarcoma vaginal se espalha por invasão local e metástase hematogênica. A idade média no diagnóstico é de cerca de 50 anos, com uma faixa que se estende desde 21 a 86 anos. $^{4}$

Uma revisão indiana de 2014 mostrou a escassez de relatos dessa patologia na literatura: Cerca de 71 casos foram relatados na literatura inglesa até a data da publicação. ${ }^{4}$

O tratamento ainda é controverso. Uma revisão de 2002, baseada em relatos de caso, mostrou que a cirurgia é utilizada como primeira escolha. ${ }^{5}$

A maioria dos casos relatados anteriormente utiliza uma abordagem cirúrgica aberta ou transvaginal para ressecção primária de tumor. A cirurgia minimamente invasiva é uma ferramenta importante na cirurgia pélvica ${ }^{6}$. Hagen et al, em 2019, relatou o sucesso de uma abordagem robótica na ressecção primária de tumores vaginais. ${ }^{6}$

Radioterapia e/ou quimioterapia podem ser úteis. Há alguns relatos em que esses tratamentos foram utilizados para diminuição do tumor e posterior abordagem cirúrgica, mas não há estudos que mostrem superioridade quando comparado a cirurgia isolada. ${ }^{7,8}$

No caso relatado, optou-se por cirurgia conservadora com exérese da massa por via vaginal e acompanhamento da paciente em regime ambulatorial. 


\section{REFERÊNCIAS}

1. Vizza E, Petrozza V, Porta N, Certelli C, Battaglione E, Corrado G, et al. Primary vaginal leiomyosarcoma: a case report with complete morphological, immunohistochemical and ultrastructural study. Taiwan J Obstet Gynecol. 2020;59(2):314-7.

2. Busca A, Parra-herran C. Myxoid mesenchymal tumors of the uterus: an update on classification, definitions, and differential diagnosis. Adv Anat Pathol. 2017;24(6):354-61.

3. Keller N, Heidi G. Leiomyosarcoma of the vagina: an exceedingly rare diagnosis. Case Rep Obstet Gynecol. 2015;2015:363895.

4. Khosla D, Patel F, Kumar R, Gowda K, Nijhawam R, Sharma S. Leiomyosarcoma of the vagina: a rare entity with comprehensive review of the literature. Int J Appl Basic Med Res. 2014;4(2):128-30.
5. Benchakroun N, Tahri Tawfig N, Acharki A, Sahrasousi S, Bender A, Kahlain A. Vaginal leyomiosarcoma, apropos of 2 cases and review of the literature. Gynecol Obstet Fertil. 2002;30(7-8):592-5.

6. Hagen J, Wilhite A, Tarbunova M, Erickson B. Successful robotic surgery for primary resection of a vaginal leiomyosarcoma: a case report. 2019;30:100503.

7. Ben Amara F, Jaouadi M, Jouini H, Nasr M, Malek M, Neji K, et al. Primary leiomyosarcoma of the vagina. Case report and literature review. Tunis Med. 2007;85:68-70.

8. Peters W, Kumar N, Andersen W, Morley G. Primary sarcoma of the adult vagina. Gynecol Oncol. 1985;20(2):253.

\section{Como citar:}

Alexandre CF, Peixoto RA, Melo KM, Cavalcante DI. Leiomiossarcoma mixoide de vagina: relato de caso. Rev Med UFC. 2021;61(1):1-4. 